\title{
DEVELOPMENT OF A NEW TIME RESOLVED IRRADIANCE UNIFORMITY MAPPER AT SPASOLAB
}

\author{
C. Barber ${ }^{(1)}$, A Martínez de Olcoz ${ }^{(2)}$, J.M. Fernández ${ }^{(2)}$, P. López ${ }^{(2)}$, G. Martínez ${ }^{(1)}$ \\ (1) INTA-SPASOLAB, Carretera de Ajalvir km. 4, 28850 Torrejón de Ardoz, Spain
}

Email:barberdcl@inta.es,martinezfg@inta.es

\begin{abstract}
(2) ISDEFE Consulting, C/ Beatriz de Bobadilla 3, 28040 Madrid, Spain,Email: martinezoam.pers_externo@inta.es, fernandezdjm.pers_externo@inta.es, lopezmp.pers_externo@inta.es,
\end{abstract}

\begin{abstract}
Irradiance uniformity mapping of solar simulators is a key characterization required for performing accurate solar cell electrical performance tests, alongside spectrum matching and stability. Within photovoltaic space standards such as [1] and [2], uniformity mapping is specified to verify the solar simulator nonconformance class. However, uniformity mappers are a relevant tool for solar simulator manufacturers as well as for space solar cell testing facilities, aiding to adjust test plane irradiance uniformity after maintenance, lamp replacement or on a more analytic approach, applying correction factors to the test plane. Thus, it is desirable to have a tool that allows for easier and faster acquisition of irradiance uniformity maps.
\end{abstract}

This paper presents a time-resolved uniformity mapper developed at Spasolab based on two main premises: easy adaptability to different solar simulators and versatility in terms of irradiance operation range, extended up to 2 AM0. A modular two-stage design features independent bias PCB's per each photodiode that allow for easy sensor swapping or different biasing for future sensor replacements. A new type of photodiode active biasing is also presented, with the main feature of being capable of switching on the bias voltage only during the acquisition process, hence reducing self-heating. Ad-hoc software developed in LabVIEW and PXI instrumentation enable the system to have different acquisition modes further expanding the irradiance range to be tested without risking hazard to the system due to overheating. Finally, outdoor calibration method and results for the system are also presented.

\section{INTRODUCTION}

The development of photovoltaics as an important power source in space missions during the 1960s brought a need to establish synthetic methods to test photovoltaic devices at array and cell level under AM0. This required a better understanding of illumination conditions that were to be recreated in order to pursue repeatability in photovoltaic characterizations throughout testing laboratories. This implied not only recreating light to match a certain spectrum and irradiance level but also to ensure that the light was shed upon the test plane in a uniform collimated fashion. As irradiance uniformity is tied to solar simulator optics and light source displacement, measurements were soon to be established as a feedback loop while developing solar simulators. In these early stages, JPL developed a test apparatus for measuring the irradiance uniformity of 15-foot Space Simulator upgrading, consisting on a single sensor swiped in polar coordinates with a reference sensor to correct stability [3], as depicted in fig. 1. This type of system is still in use and while benefiting from taking data from only one sensor, corrections with the second sensor must be applied and the automated sweep is normally time-consuming.

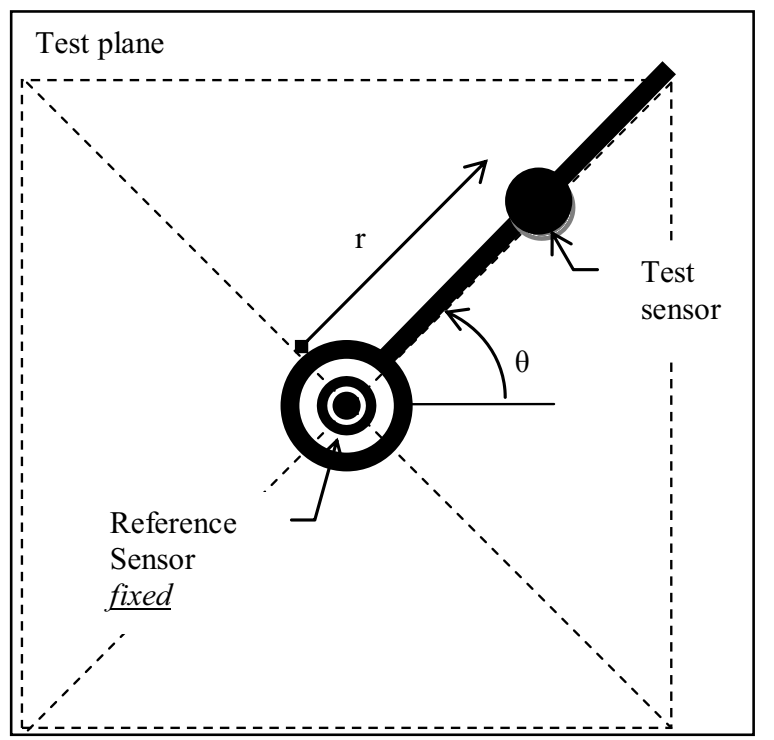

Figure 1 Diagram of a polar sweep single sensor uniformity mapper 
With advances in instrumentation and data acquisition, more affordable approaches for a multiple sensor simultaneously acquired systems have been made available. In the past years, the development of these time resolved multiple sensor uniformity mapping systems have been published both for large area flash simulators and for steady state such as in [4]. Such systems present an array of sensors that are synchronized and simultaneusly acquired, hence, obtaining irradiance maps quickly and rejecting light source instabilities. Such system is depicted in fig. 2 .

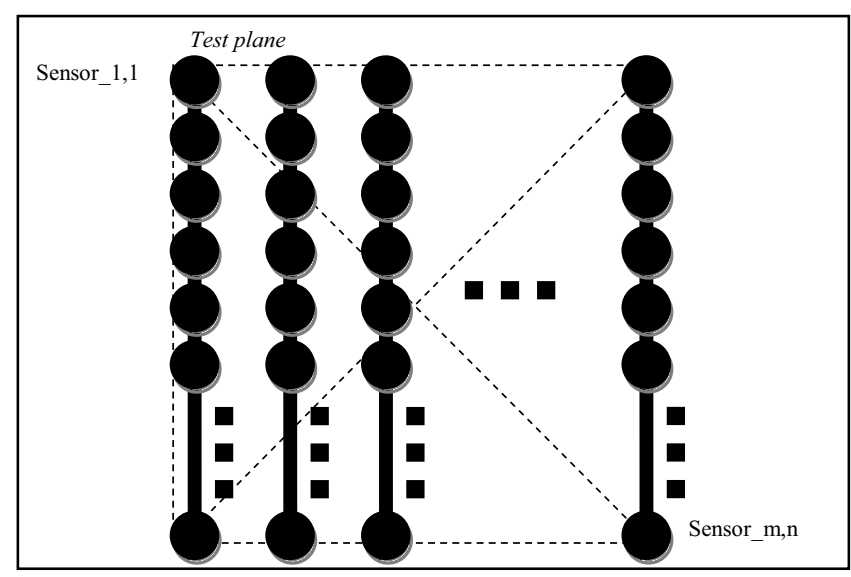

Figure 2 Two dimension sensor array uniformity mapper diagram

However, these types of systems have plenty of room for different strategies and developments yet. In this paper we will present the developed time resolved uniformity mapper at Spasolab, focused on steady state solar simulators but with potential capabilities for performing under flash systems. Special attention has been placed on having a wide irradiance linearity response from the sensors, surpassing 2 AM0. Also, effective sensor area and spectral response makes the system suitable to comply with [1] as well as other terrestrial standards.

\section{HARDWARE DEVELOPMENTS}

\subsection{Overview}

A two stage approach has been taken for developing the printed circuit board (PCB) harness. Up to 40 photodiodes, of $1 \mathrm{~cm}^{2}$ effective area, can be housed in the main printed circuit board. The design currently includes high quality silicon photodiodes with quartz cover. Sensor deployment covers an area of 146 by 146 $\mathrm{mm}$, placed in a checkered pattern (subsequent rows of 4 and 5 sensors). This pattern presents a uniform distribution, without weighing specific areas. Sensors are mounted on their individual pcb's which harbour the bias circuit and are connected to the main PCB through polarised flat 9 pin connectors. This allows for quick replacement, and for various configurations.

The hardware is completed with a custom manufactured aluminium chassis that also houses the connectors and led indicators. A window lid has also been developed to prevent irradiation from hitting the main PCB and from undesired reflexions from the coating. This lid will also enforce flatness throughout the sensors, as interconnectors can wobble. The chassis and the system's compact form enables easy mounting on various tests platforms in vertical or horizontal orientation, even being capable of placement within a testing chamber.

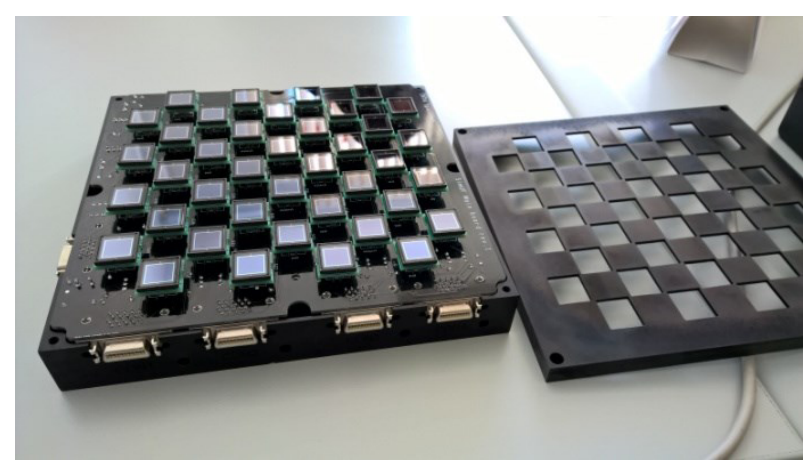

Figure 3 A complete view of the system, including bias $P C B$ with sensors mounted on the main PCB. Custom chassis and top window lid also shown.

\subsection{Biasing Circuit}

A simple active bias circuit that hasn't been found within the literature has been developed. Requiring negative voltage bias to further increase the photodiode's linear operation, a simple option employing a single small profile integrated circuit has been used, which is a low voltage dropout linear regulator (LDO). These small footprint voltage regulators present a controlled voltage output, presenting low voltage drop with load current. A common feature nowadays is the addition of enable pins, that allow fast transitions from on (regulated voltage out) and off (high impedance) states. For this application, a CMOS based $3.3 \mathrm{~V}$ regulator from Microchip, the TC1185, has been employed. The developed topology can be seen in fig. 4. Having the capability of disconnecting the biasing voltage is of great value, avoiding self-heating of the devices due to power consumption. 


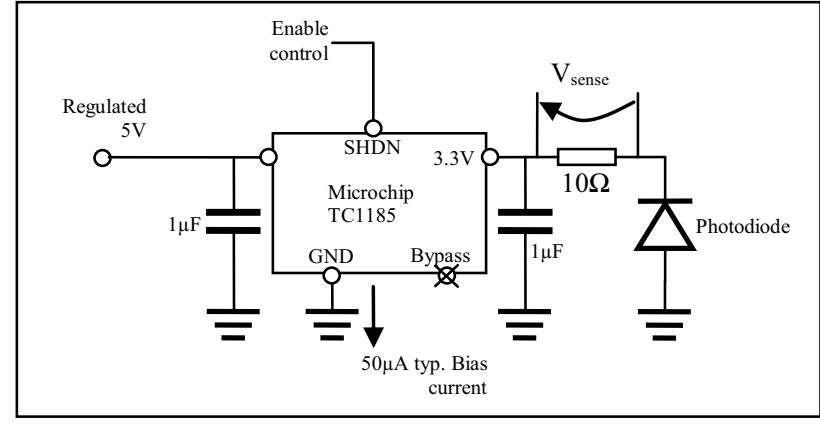

Figure 4 Developed bias circuit with Microchip's TC1185

The current loading capabilities of the LDO are $150 \mathrm{~mA}$, which presents enough headroom for the expected photodiode current, rated in the vicinity of $40 \mathrm{~mA}$ for AM0. This topology presents some inconveniences when compared to transimpedance topologies, such as not being able to include a gain stage or having less bandwidth. For the latter, bandwidth is not an issue in steady state simulators where xenon flicker components fall under $1 \mathrm{KHz}$ and it is yet to be tested under flash simulators. Despite not having a gain stage, a greater resistor for the voltage readout can be introduced, in the present case being a $10 \Omega$ precision resistor.

Taking the photodiodes response and the output behaviour of the LDO as shown in the manufacturer's datasheet, and also taking into account the voltage drop in the test resistor, an operation value of 2 AM0 has been calculated to be within safe operation boundaries, both of the photodiode presenting linear response as for the LDO.

The LDO has a typical rise time of under $200 \mu$ s when activating through the enable pin. This has been monitored in 0 load, however, the software enables the circuit $50 \mathrm{~ms}$ prior to the data acquisition. Therefore, no transients have been observed at the tested irradiance levels, neither after increasing acquisition rates up to $100 \mathrm{Ks} / \mathrm{s}$.

Circuit layout with the sensor mounted can be seen in fig. 5 and fig. 6.

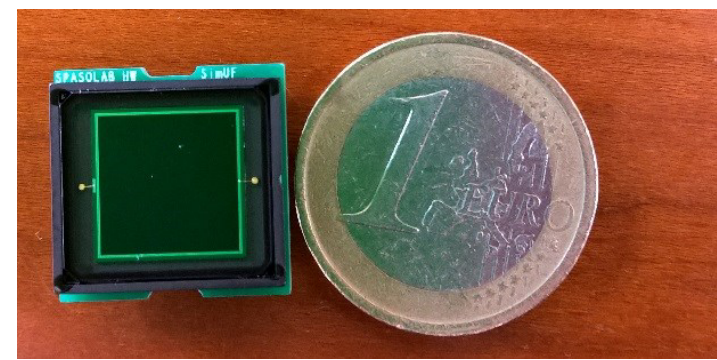

Figure 5 Front view of the silicon photodiode mounted on the bias $P C B$.

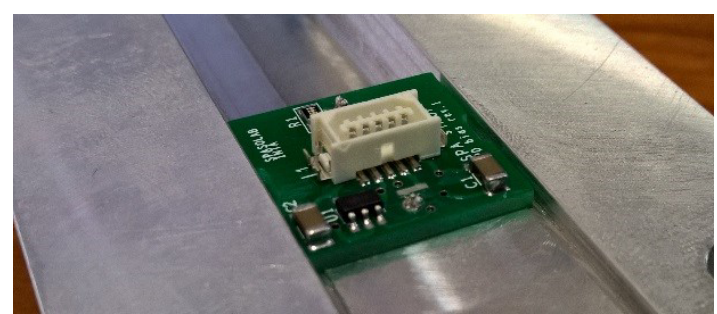

Figure 6 Rear view of the bias PCB showing the interconnector and the low dropout voltage regulator.

\subsection{Data Acquisition and control software}

The system has been based in a PXI unit with five simultaneous acquisition 8 analogue channel boards from National Instrument's $\mathrm{S}$ series. High data throughput is available, and acquisition rates can be set to 100Kilosamples/second simultaneously and synchronized throughout all 40 channels. A bespoke software application has been developed in LabVIEW. The software controls data acquisition, on state timing and raw data management. As mentioned earlier, software only activates bias voltage to the photodiodes during acquisition, avoiding uncontrolled temperature increase due to the photodiode's power consumption. In idle state the sensor is biased at $0 \mathrm{~V}$.

Taking advantage of the versatility offered by the PXI unit, three operation modes have been developed:

- Normal operation: Measurement is triggered on demand, activating LDO only during acquisition time.

- Continuous operation: In this mode the software triggers measurements until stopped by the user. With the $3 \mathrm{D}$ graphic view of the irradiance map, this mode enables for a near real time view of the light uniformity.

- Light Triggered: This mode triggers the acquisition when light is shed upon the device. This mode allows measuring higher irradiances without incurring in an uncontrolled temperature increase to hazardous levels as well as maximizing temperature uniformity. This allows for stabilization across sensors at laboratory conditions prior to the test.

\section{CALIBRATION}

\subsection{Linearity Test}

Prior to outdoor calibration, an indoor linearity test has been performed employing the light superposition method [5]. To do so, a halogen lamp ring placed on one of Spasolab's solar simulator facilities has been employed. The halogen ring is comprised by 6 lamps, 
all unfiltered, and at $75 \%$ nominal power. These have been separated into three groups (LA,LB,LC), conforming a total of 4 irradiance steps.

Table 1 Superposition analysis lamp step configuration

\begin{tabular}{|c|c|}
\hline Measured & Summed \\
\hline LALB & LA+LB \\
\hline LALC & LA+LC \\
\hline LBLC & LB+LC \\
\hline LALBLC & LA+LB+LC \\
\hline
\end{tabular}

Raw data has been plotted for each step, and is shown in separate 3D maps for a qualitative perspective on the contributions of each lamp configuration, which is shown in fig. 7. Vertical unit is taken directly from the raw data, and is expressed as the voltage measured across the precision resistance.

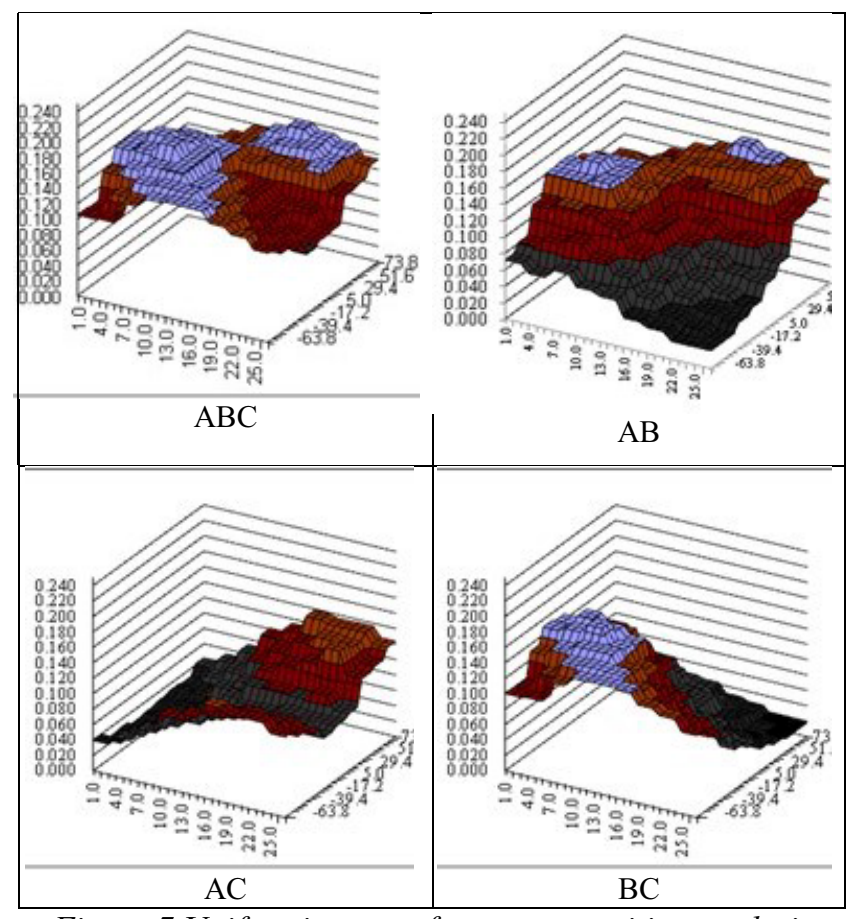

Figure 7 Uniformity maps from superposition analysis

Data later has been analyzed, evaluating the sum of individual sources and comparing it to the measured value. Data fitting has been evaluated using least squares method. Slope and coefficient of determination (R2) factor have been condensed in fig. 8 and in fig. 9 . Sensors positions 12 and 14 have been omitted due to an issue with the acquisition channel and failure in the cable harness respectively.

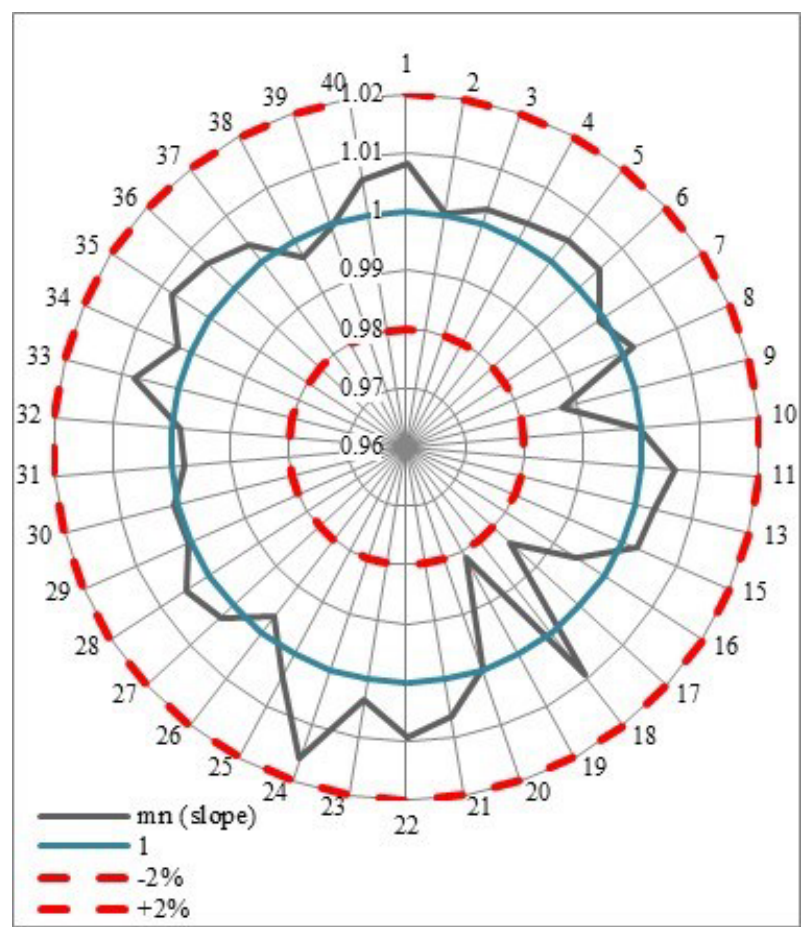

Figure 8 Radar chart with linear estimation of m(slope) employing superposition analysis

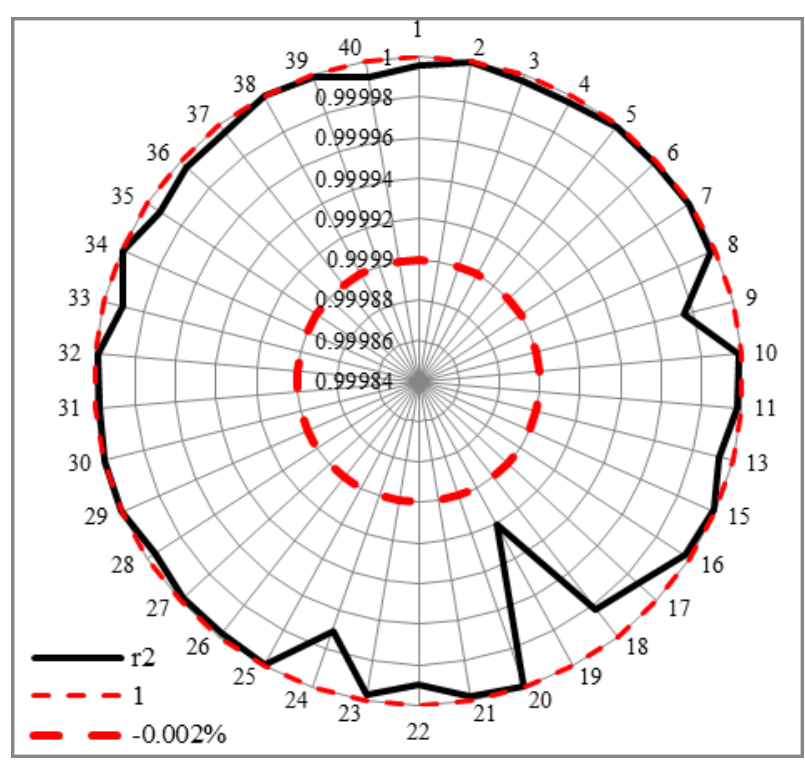

Figure 9 Radar chart with R2 from the linear estimation of the superposition analysis

Data fitting has shown excellent results for the linearity of the complete system. It is interesting to observe the high match for the $\mathrm{R} 2$ of each position.

Summing up what this test has yielded; linearity of the complete system has been evaluated and proved to have a linear response throughout the signal chain. Unfortunately, this analysis will have to be repeated 
with other light configuration capable of injecting a response of up to 1.5AM0 equivalent irradiance. The current setup has been chosen due to the high stability of halogen lamps power by precision power sources. However, this paves the way to establish an easier calibration map for the device.

\subsection{Outdoor calibration}

Outdoor calibration has been performed with the premise that irradiance throughout the device is ideally uniform. The configuration for the test is comprised by the uniformity mapper, a calibrated pyranometer and a primary working standard solar cell. At the time of the test, the elevation angle was adjusted by the use of the primary standard, fixing the structure at its maximum short circuit current Isc. The aim is to achieve maximum uniformity of light. At the time of the tests, sky was clear, GNI was greater than $1000 \mathrm{w} \cdot \mathrm{m}^{-2}$ with a variation of less than $2 \%$ in any 5 minute interval. No visible clouds or hazy conditions in $45^{\circ}$ view angle around the sun. The wind speed was also under $2 \mathrm{~m} / \mathrm{s}$, hence, temperature is considered uniform throughout the device.

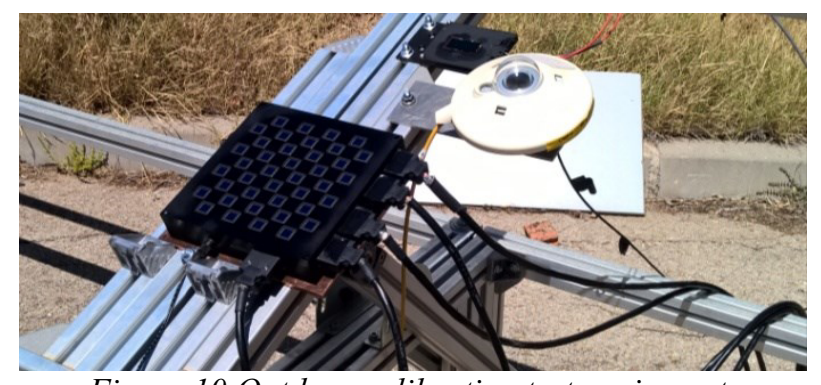

Figure 10 Outdoor calibration test equipment displacement

Two tests are compared. The first will include data that is employed to create a calibration matrix by normalizing all the sensors with respect to sensor 16 , which has been chosen due to its centred position as well as for its good coefficient of determination and slope in the linearity analysis. Software has been configured to perform tests with $1 \mathrm{KHz}$ sampling rate and a total of 1000 samples. Triggering method is set to normal (triggers when launched).

The irradiance value measured with the piranometer at the time of each test is shown in tab. 2 .

Table 2 Irradiance levels at the time of the tests

\begin{tabular}{|c|c|}
\hline Test 1. Calibration & $\begin{array}{c}\text { Test } 2 \text {. Compensating with } \\
\text { calibration matrix }\end{array}$ \\
\hline $1004.873 \mathrm{~W} \cdot \mathrm{m}-2$ & $1005.994 \mathrm{~W} \cdot \mathrm{m}-2$ \\
\hline
\end{tabular}

As mentioned the calibration matrix is obtained by normalizing with respect to sensor position 16 . This matrix is stored and new uniformity values are compensated using the relation in Eq. 1.

$$
S_{i}^{*}=\frac{S_{i}}{c m_{i}} \rightarrow \text { value in } m V
$$

Where $\mathrm{S}^{*}$ is the compensated value at position $\mathrm{i}, \mathrm{S}$ the raw data input at position $\mathrm{i}$ and $\mathrm{cm}$ the compensation matrix. For data treatment and representation, sensor positions 12 and 14 have been removed as aforementioned. For representation purposes, gaps between sensors have been filled by averaging the surrounding sensor values, which doesn't modify non conformance calculation, since maximums and minimums are left untouched. The uniformity map of "Test 2" without compensation and after compensating with the normalized matrix from test 1 , is shown in fig. 11 and fig. 12 respectively. The scale shown is centimetres for the plane and volts for the sensor output level.

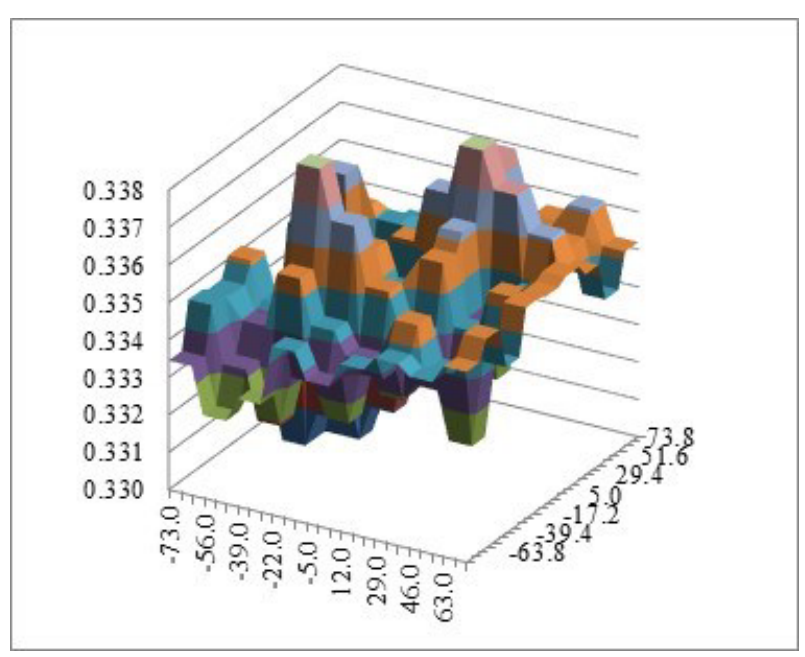

Figure 11 Test data without compensating values

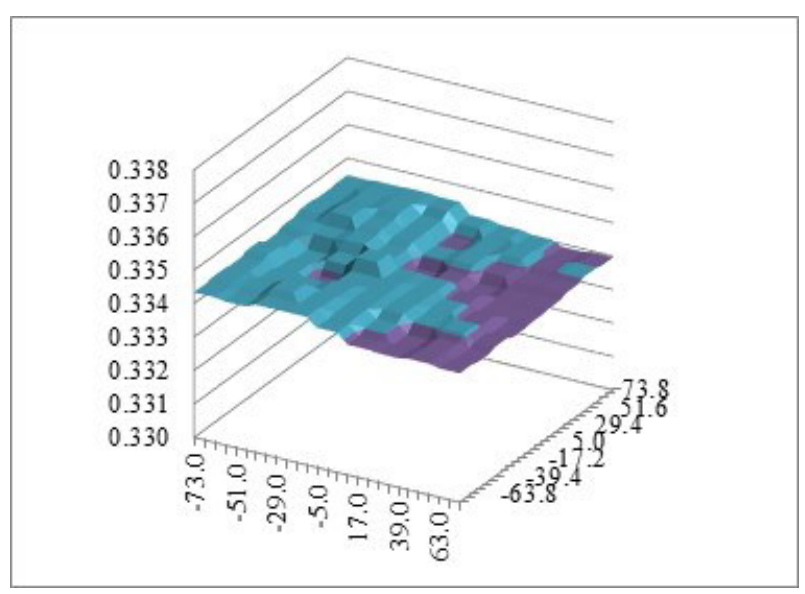

Figure 12 Test Data after compensation 
The non conformance values for each non compensated and compensated uniformity maps are shown in tab. 3 .

Table 3 Non conformance values with and without

compensation of Test 2 throughout the test plane

\begin{tabular}{|c|c|}
\hline $\mathrm{NC}(\%)$ w/o compensation & $\mathrm{NC}(\%)$ with compensation \\
\hline 3.551 & 0.134 \\
\hline
\end{tabular}

This shows clearly the data dispersion straight out of the raw data file must be compensated. A valid compensation matrix should be averaged over a higher number of test samples and for different uniform intensities. However, at the time of edition of this paper, test data was not available.

\section{CONCLUSIONS AND FUTURE WORK}

This paper presents a versatile and high linear range photodiode based time resolved uniformity mapper developed at SPASOLAB. Novel features include a new and simple active bias topology that allows disabling bias voltage to prevent the photodiode from consuming power while in idle, enabling bias voltage only during acquisition. The biasing circuit along with the photodiodes have been developed to test uniformity maps up to 2AM0, although such tests are not included within this work. A modular approach allowing quick sensor swapping and a bespoke compact aluminium chassis enhances versatility for performing irradiance uniformity measurements to a wide variety of solar simulators. Custom developed LabVIEW software, along with PXI instrumentation, provides different modes of operation that can trigger tests automatically with light.

Future works comprehend a tighter characterization of the device, exposing it to different light sources and configurations, and performing an adequate uncertainty analysis. Swapping silicon for infrared photodiodes for longer wavelength uniformity mapping would also be of interest. The verification of the system's capabilities to test irradiance uniformity maps of ultra violet simulators is also in sight.

\section{ACKOWLEDGEMENTS}

The research was co-supported by the European Space Agency (ESA) under contract 4000107040/12/NL/GLC and the European Metrology Research Programme under the project ENG 55 (Photoclass). The EMRP is jointly funded by the EMRP participating countries within EURAMET and the European Union.

\section{REFERENCES}

1. European Cooperation for Space Standarization (2012), "ECSS-E-ST-08 "Space Enineering. Photovoltaic assemblies and components"," ESA, Nordwijk ZH.

2. ISO (2005), "ISO15387-05. Space Systems Single-junction solar cells - Measurement and calibration procedures," ISO.

3. NASA (1968), "Design and Construction of the 15-ft-Beam Solar Simulator SS15B," NASA, Pasadena, CA.

4. D. Rivola, S. Dittman, M. Pravettoni, G. Friesen and D. Chianese (2014), "High-Speed Multi-Channel System for Solar Simulator Irradiance Non-Uniformity Measurment," Photovoltaic Specialist Conference (PVSC), IEEE 40th.

5. K. Emery, S. Winter, S. Pinegar and D. Nalley (2006), "Linearity Testing of Photovoltaic Cells," in 4th World Conference on Photovoltaic Energy Conversion (WCPEC-4) IEEE, Waikoloa. 